\title{
Télescope
}

Revue d'analyse comparée en administration publique

\section{Understanding E-Government in Europe: Issues and Challenges, sous la direction de Paul G. Nixon, Vassiliki N. Koutrakou et Rajash Rawal, London, Routledge, 2010, 322 p.}

\section{Mathieu Tremblay}

Volume 18, numéro 1-2, printemps-été 2012

URI : https://id.erudit.org/iderudit/1009264ar

DOI : https://doi.org/10.7202/1009264ar

Aller au sommaire du numéro

Éditeur(s)

L'Observatoire de l'administration publique

ISSN

1203-3294 (imprimé)

1929-3348 (numérique)

Découvrir la revue

Citer ce compte rendu

Tremblay, M. (2012). Compte rendu de [Understanding E-Government in Europe: Issues and Challenges, sous la direction de Paul G. Nixon, Vassiliki N. Koutrakou et Rajash Rawal, London, Routledge, 2010, 322 p.] Télescope, 18(1-2), 207-209. https://doi.org/10.7202/1009264ar d'utilisation que vous pouvez consulter en ligne.

https://apropos.erudit.org/fr/usagers/politique-dutilisation/ 


\title{
Recensions
}

\section{UNDERSTANDING E-GOVERNMENT IN EUROPE: ISSUES AND CHALLENGES}

Sous la direction de Paul G. Nixon, Vassiliki N. Koutrakou et Rajash Rawal, London, Routledge, 2010, 322 p.

\begin{abstract}
$\mathrm{A}$ u cours des dernières années, la démocratisation à grande échelle des technologies de l'information et de la communication (TIC) a poussé les États à investir massivement dans le développement et la mise en œuvre de systèmes de gouvernement en ligne (e-government). Les objectifs poursuivis par les gouvernements qui s'engagent dans cette voie sont multiples.

D'abord, dans un contexte budgétaire difficile, les TIC renferment un énorme potentiel de réduction des coûts par l'accroissement de l'efficacité dans le traitement des tâches et des opérations administratives. Les TIC sont également considérées comme un outil permettant d'offrir de meilleurs services centrés sur le client, plutôt qu'en fonction de la structure administrative décentralisée de plusieurs administrations publiques. Troisièmement, les gouvernements en ligne détiennent le potentiel de rehausser la transparence gouvernementale et le lien de confiance avec les citoyens. Les initiatives de gouvernement ouvert, par lesquelles les organisations publiques partagent de façon proactive leurs données avec les citoyens par l'entremise d'applications Internet, accroissent leur transparence alors que les nouveaux outils du Web 2.0, tels que les médias sociaux, favorisent l'engagement et la participation citoyenne d'une façon inédite. Enfin, un objectif plus ambitieux consiste à s'appuyer sur les TIC pour moderniser et transformer l'administration publique. Habituées à travailler en silo, les différentes unités administratives, afin de réduire leurs coûts de fonctionnement et de centrer les programmes et les services sur les clients, doivent changer leur façon de concevoir la fonction publique et intensifier leur collaboration et le partage de leurs données tout en respectant les dispositions légales concernant la confidentialité et la sécurité des renseignements personnels de leurs clients. Transformer la culture organisationnelle de structures administratives aussi complexes constitue un défi de taille et les TIC en sont souvent la pierre angulaire.

Si la vaste majorité des gouvernements a investi à différentes échelles dans le développement de services en ligne, la recherche scientifique sur les apports de cette stratégie en matière de gouvernance s'avère plutôt récente. C'est dans ce contexte que l'ouvrage collectif intitulé Understanding E-Government in Europe: Issues and Challenges et publié chez Routledge constitue une avancée significative, tant sur le plan de la réflexion théorique que des études de cas empiriques qu'il rassemble. Dirigé par Paul G. Nixon (The Hague University - Pays-Bas), Vassiliki N. Koutrakou
\end{abstract}


(University of East Anglia - Royaume-Uni) et Rajash Rawal (The Hague University - Pays-Bas), il réunit les contributions de trente professeurs et chercheurs européens spécialisés dans les politiques publiques et la technologie. Non seulement cet ouvrage évalue-t-il, à partir d'expériences européennes, le potentiel des TIC pour faciliter l'adoption de nouvelles formes de gouvernance et de participation, mais il tente également de répondre à de nombreuses questions d'ordre politique et organisationnel liées à l'utilisation des nouvelles technologies par les administrations publiques : est-ce que la société provoque les initiatives de gouvernement en ligne ou y répond-elle? Est-elle apte à s'y adapter? Quelles sont les conséquences des gouvernements en ligne sur la relation entre le pouvoir et la démocratie? La technologie est-elle suffisamment avancée pour une utilisation optimale des gouvernements en ligne? Comment le gouvernement en ligne est-il perçu par la population? Comment gérer les questions délicates liées à l'identité, à la protection des renseignements personnels et à l'inclusion sociale? Comment traiter les enjeux d'activisme, de cyberterrorisme, de biométrie et de relations internationales à l'ère numérique? Voilà quelques-uns des nombreux thèmes abordés par les dix-sept chapitres de ce riche ouvrage.

Les deux premières parties du collectif posent les éléments fondamentaux et les principaux défis conceptuels qui entourent la notion de gouvernement en ligne. Il est question, entre autres, de débats théoriques entourant Internet et les TIC en tant que " cinquième pilier " d'autorité institutionnelle de l'État moderne; des facteurs de succès et d'échec dans le déploiement d'initiatives de gouvernement en ligne; des possibilités de transformation de la fonction publique par l'entremise du gouvernement en ligne; des notions d'offre et de demande pour de tels services; du fossé entre la théorie sur la cyberdémocratie et la pratique de la participation citoyenne dans un contexte technologique; du bilan mitigé des consultations en ligne; du concept de reddition de comptes dans le cadre du cybergouvernement. Souvent basées sur des expériences concrètes, ces contributions proposent des réflexions théoriques et conceptuelles critiques sur les principales notions entourant le gouvernement en ligne.

Dans la troisième partie de l'ouvrage, les auteurs se concentrent sur des thèmes précis en s'appuyant généralement sur une étude de cas européenne. Par exemple, Savirimuthu décrit les apports potentiels des technologies de l'information et des médias sociaux dans l'administration de la justice européenne (e-justice), notamment en ce qui a trait aux mécanismes de résolution des conflits dans le cadre de la Cour européenne des droits de l'homme. Pour leur part, Lips, Taylor et Organ explorent, à partir du cas britannique, l'un des principaux défis liés au développement des services gouvernementaux en ligne : la gestion électronique des identités des utilisateurs et des prestataires de services. Savvides et Koutrakou s'intéressent quant à eux aux facteurs de succès dans la mise en œuvre d'un système de santé électronique et Misuraca, Rossel et Glassey décrivent la performance du gouvernement en ligne tel qu'il est développé en Suisse par les différentes innovations organisationnelles préalablement instituées au sein des ministères et des organismes publics. Enfin, dans une étude comparée de cas néerlandais, britannique et norvégien, Edward évalue le rôle clé des politiciens, par leur propre utilisation des 
nouveaux outils de communication, dans le développement et l'évolution de ce qu'il qualifie de cyberdémocratie.

Composée de quatre chapitres, la dernière partie traite des relations entre le cybergouvernement et les différents acteurs. Ainsi, Nixon, Grebner et Sudulich analysent les nouvelles interactions entre le gouvernement et les organisations non gouvernementales qui résultent de l'établissement du gouvernement en ligne, particulièrement des outils du Web 2.0. Rawal évalue pour sa part les nouvelles menaces liées au cyberterrorisme et s'interroge sur les mesures prises par les gouvernements pour y répondre. Par la suite, Nixon étudie, en s'appuyant sur le cas britannique, le lien existant entre le niveau de confiance des citoyens envers le gouvernement en ligne et la couverture médiatique qui en est faite. Enfin, dans le dernier chapitre, Koutrakou explore le potentiel (et les défis) qu'offrent les TIC et les initiatives de cybergouvernement comme outils de communication au sein des organisations régionales et entre les organisations intergouvernementales multilatérales.

Par la richesse des sujets traités et des questionnements théoriques soulevés, Understanding e-Government in Europe constitue une contribution significative et attendue à la recherche sur cette tendance irréversible vers le développement de gouvernements en ligne. Ce phénomène a transformé - et continuera de le faire - la façon dont les citoyens interagissent avec leurs gouvernements. Ainsi, cet ouvrage multidisciplinaire intéressera les chercheurs et les praticiens d'une multitude de domaines : politiques publiques, gestion, science politique, communication et médias, sociologie et droit.

Par MATHIEU TREMBLAY

Professionnel de recherche au Laboratoire d'étude sur les politiques publiques et la mondialisation de l'École nationale d'administration publique du Québec et Doctorant à I'Institut québécois des hautes études internationales de I'Université Laval 